\title{
VALUE DISTRIBUTION OF LINEAR COMBINATIONS OF AXISYMMETRIC HARMONIC POLYNOMIALS AND THEIR DERIVATIVES
}

\author{
Peter A. McCoy
}

\begin{abstract}
In this paper the geometry of the value distribution of linear combinations of axisymmetric harmonic polynomials (AHP) and their derivatives is studied using the Bergman integral operator method and methods from the analytic theory of polynomials. For a given AHP, zero free cones in $E^{3}$ can be determined which are stationary for specified classes of these linear combinations in the sense that the given AHP describes cones which have an empty intersection with the level sets of all linear combinations from each class.
\end{abstract}

In addition to these results, an AHP analog to the classical theorem of Lucas' is obtained. The above results are extended to generalized axisymmetric harmonic polynomials by an operator due to R. P. Gilbert.

The study of the value distribution of AHP by the Bergman method was initiated by Morris Marden [4]. In that paper, the Bergman method [1] was used to transform polynomials of one complex variable into AHP. Methods from the analytic theory of polynomials were used with operator to obtain theorems on the location of sets in $E^{3}$ where an AHP omits a given complex value. These results were specified in terms of a pair of cones in $E^{3}$ which were functions of the convex hull of the zero set of a polynomial of one complex variable associated with the AHP by the Bergman operator.

Let $(x, y, z)$ be rectangular coordinates and $(x, \rho, \phi)$ be cylindrical coordinates with

$$
\rho^{2}=y^{2}+z^{2}, y=\rho \cos \phi, z=\rho \sin \phi .
$$

A function is said to be axisymmetric if it is independent of $\phi$.

Every AHP can be represented by the Bergman method as the integral transform of a polynomial of one complex variable (see [4]) which shall be referred to as the associate of the AHP. That is, if $H(x, \rho)$ is an AHP then there is a unique polynomial $h(\zeta)$ of the complex variable $\zeta$ such that

$$
H(x, \rho)=\frac{1}{2 \pi} \int_{0}^{2 \pi} h(x+i \rho \cos t) d t .
$$

In addition, each AHP can be represented in the form (see [3]) 


$$
H(x, \rho)=\sum_{k=0}^{n} a_{k} r^{k} P_{k}(\cos \theta)
$$

where $P_{k}(v)$ is the Legendre polynomial of degree $k$ in $v$ and $(r, \theta, \phi)$ are the spherical coordinates

$$
\rho=r \sin \theta \text { and } x=r \cos \theta .
$$

Let $C$ denote the complex plane, $K$ a compact subset of $C, \bar{K}$ the reflection of $K$ in the $x$-axis and $\mathscr{H}[K]$ the convex hull of $K$. The points on the $x$-axis to the right and left of $\mathscr{H}[K \cup \bar{K}]$ for which $\mathscr{H}[K \cup \bar{K}]$ subtends a given angle $2 \theta, 0<\theta \leqq \pi / 2$, are denoted by $\alpha_{0}=\alpha_{0}(K)$ and $\alpha_{1}=\alpha_{1}(K)$ respectively. The sectors defined by

$$
j \pi-\theta<\arg \left(\zeta-\alpha_{j}\right)<\theta+j \pi, 0 \leqq j \leqq 1
$$

are denoted by $S_{R}(K, \theta)$ and $S_{L}(K, \theta)$ respectively and the sectors opposite these, $S_{L}^{0}(K, \theta)$ and $S_{R}^{0}(K, \theta)$, are determined by a rotation of $S_{L}(K, \theta)$ and $S_{R}(K, \theta)$ about their vertices thru an angle of $\pi$.

Let $0<\gamma, \delta<\pi / 2$ and $C_{R}(K, \gamma)$ represent the cone

$$
0 \leqq \rho<\left(x-\alpha_{0}\right) \tan \gamma
$$

and $C_{L}(K, \delta)$ the cone

$$
0 \leqq \rho<\left(\alpha_{1}-x\right) \tan \delta
$$

For $\kappa$ real and $0<\beta \leqq \pi$, let $S_{\kappa}(\beta)$ denote the sector

$$
\kappa-\beta<\arg \zeta<\beta+\kappa
$$

The points at which a polynomial of one complex variable assumes the complex value $\alpha$ shall be referred to as the $\alpha$ points of the polynomial. We shall deal with AHP having degree at least 2.

THEOREM 1. Let $\alpha \in C, H$ be an AHP of degree $n$ and $K$ denote the convex hull of the $\alpha$ points of the associate of $H$. Then for each $\lambda=\left\{\lambda_{1}, \cdots, \lambda_{p}\right\} \subset S_{L}(K, \pi / 2 n) \cap S_{\pi}(\pi / 2 n)$ with $1 \leqq p \leqq n$ the AHP

$$
G_{p}(\lambda) H=\left(1-\lambda_{1} \frac{\partial}{\partial x}\right) \cdots\left(1-\lambda_{2} \frac{\partial}{\partial x}\right) \cdots\left(1-\lambda_{p} \frac{\partial}{\partial x}\right) H
$$

omits $\alpha$ in $C_{R}(K, \pi / 2 n)$. For each $\mu=\left\{\mu_{1}, \cdots, \mu_{p}\right\} \subset S_{R}(K, \pi / 2 n) \cap$ $S_{0}(\pi / 2 n)$, the $A H P$

$$
G_{p}(\mu) H=\left(1-\mu_{1} \frac{\partial}{\partial x}\right)\left(1-\mu_{2} \frac{\partial}{\partial x}\right) \cdots\left(1-\mu_{p} \frac{\partial}{\partial x}\right) H
$$

omits $\alpha$ in $C_{L}(K, \pi / 2 n)$. 
Proof. Let the associate of $H$ be $h$ and

$$
\left\{\lambda_{1}, \cdots, \lambda_{p}\right\} \subset S_{L}(K, \pi / 2 n) \cap S_{\pi}(\pi / 2 n) .
$$

Consider the AHP,

$$
G_{p}(\lambda) H=\left(1-\lambda_{1} \frac{\partial}{\partial x}\right) \cdots\left(1-\lambda_{p} \frac{\partial}{\partial x}\right) H
$$

which may be written in terms of its associate as

$$
G_{p}(\lambda) H=\frac{1}{2 \pi} \int_{0}^{2 \pi} g(x+i r \cos t) d t
$$

with

$$
g(z)=\left(1-\lambda_{1} \frac{d}{d z}\right) \cdots\left(1-\lambda_{p} \frac{d}{d z}\right) h(z) .
$$

By a theorem of Takagi [5, p. 83], the zero set of $g(z)-\alpha$ lies in the convex hull of the region $A$ swept out by translating the zero set of $h(z)-\alpha, K$, by amounts $n \lambda_{1}, n \lambda_{2}, \cdots$ and $n \lambda_{p}$. But $\left\{n \lambda_{1}, n \lambda_{2}, \cdots\right.$, $\left.n \lambda_{p}\right\} \subset S_{L}(K, \pi / 2 n) \cap S_{\pi}(\pi / 2 n)$ which implies that $A \subset S_{R}^{0}(K, \pi / 2 n)$.

We factor $g(z)-\alpha$ as

$$
g(z)-\alpha=A_{n}(\lambda) \prod_{j=1}^{n}\left(z-\xi_{j}(\lambda)\right)
$$

and choose $x=x_{0}$ and $\rho=\rho_{0}$ as the equations of a circle in $C_{R}(K, \pi / 2 n)$. Then the vector $z=z(t)=x_{0}+i \rho_{0} \cos t$ terminates in $S_{R}(K, \pi / 2 n)$ for $0 \leqq t<2 \pi$. As

$$
\left\{\xi_{1}(\lambda), \cdots, \xi_{n}(\lambda)\right\} \subset S_{R}^{0}(K, \pi / 2 n),
$$

we find that for $0 \leqq t \leqq 2 \pi$ and $1 \leqq j \leqq n$,

$$
-\pi / 2 n<\arg \left(z(t)-\xi_{j}(\lambda)\right)<\pi / 2 n
$$

which implies that

$\arg A_{n}(\lambda)-\pi / 2<\arg (g(z(t))-\alpha)<\arg A_{n}(\lambda)+\pi / 2$ for $0 \leqq t \leqq 2 \pi$.

The integrand of (3) considered as the limit of a sum of vectors terminating in an open half plane with boundary through the origin cannot vanish, thus $G_{p}(\lambda) H \neq \alpha$ in $C_{R}(K, \pi / 2 n)$ as was to be proved. The remaining case is shown in a similar manner.

CoRollary 1.1. Let $\alpha \in \boldsymbol{C}, H$ be an AHP of degree $n$ and $K$ denote the convex hull of the $\alpha$ points of its associate. Let $1 \leqq k \leqq$ $n$ and 


$$
G_{k}(\nu) H=\sum_{j=0}^{k} c(k, j)(-1)^{j} \nu^{j}\left(\frac{\partial}{\partial x}\right)^{j} H, c(k, j)=k ! / j !(k-j) ! .
$$

Then for each $\eta \in S_{L}(K, \pi / 2 n) \cap S_{\pi}(\pi / 2 n)$,

$$
G_{k}(\eta) H \neq \alpha, 1 \leqq k \leqq n
$$

in $C_{R}(K, \pi / 2 n)$ and for each $\nu \in S_{R}(K, \pi / 2 n) \cap S_{0}(\pi / 2 n)$,

$$
G_{k}(\nu) \neq \alpha, 1 \leqq k \leqq n
$$

in $C_{L}(K, \pi / 2 n)$.

Proof. We observe that Theorem 1.1 may be applied to

$$
G_{k}(\lambda) H-\alpha=\left(1-\eta \frac{\partial}{\partial x}\right)^{k}(H-\alpha)
$$

with $\lambda=\{\eta, \cdots, \eta\}$

Corollary 1.2. Let $\alpha \in C, H$ be an AHP of degree $n$ and $K$ be the convex hull of the $\alpha$ points of its associate. Let

$$
G_{k}(\nu) H=\left(1-\nu_{1} \frac{\partial}{\partial x}\right) \cdots\left(1-\nu_{p} \frac{\partial}{\partial x}\right)\left(\frac{\partial}{\partial x}\right)^{k} H
$$

with $1 \leqq p+k \leqq n$ and $\nu=\left\{\nu_{1}, \nu_{2}, \cdots, \nu_{n}\right\}$. Then for each $\lambda=\left\{\lambda_{1}, \cdots\right.$, $\left.\lambda_{p}\right\} \subset S_{L}(K, \pi / 2 n) \cap S_{\pi}(\pi / 2 n)$,

$$
G_{k}(\lambda)(H-\alpha) \neq 0,1 \leqq p+k \leqq n
$$

in $C_{R}(K, \pi / 2 n)$ and for each $\mu=\left\{\mu_{1}, \cdots, \mu_{p}\right\} \subset S_{R}(K, \pi / 2 n) \cap S_{0}(\pi / 2 n)$

$$
G_{k}(\mu)(H-\alpha) \neq 0
$$

in $C_{L}(K, \pi / 2 n)$.

Proof. We observe that as $K$ is the convex hull of the $\alpha$ points of the associate of $H, h$, Lucas' theorem [5, p. 22] implies that $K$ contains the zeros of $(h-\alpha)^{(k)}$ for $1 \leqq k \leqq n-1$. The result follows by applying Theorem 1 to the AHP $(\partial / \partial x)^{k}(H-\alpha)$.

Let $A \subset \boldsymbol{C}$ and $\gamma \in \boldsymbol{C}$. The translate of $A$ by the vector $\gamma$ shall be denoted by $A(\gamma)$.

Lemma 1. Let $\alpha \in C, f$ be a polynomial of degree $n$ and $K$ denote the convex hull of the $\alpha$ points of $f$. Let

$$
L_{j}(\nu) f=f-\nu^{2^{j}} f^{\left(2^{j}\right)} \quad 0 \leqq j \leqq k
$$

where $k$ is the largest integer such that $2^{k} \leqq n$ and $\nu \in S_{R}(K, \pi / 2 n) \cap$ 
$S_{0}\left(\pi /(2 n)^{2}\right)$. Then for any solution $\nu_{\circ}$ to the equation

$$
\arg \left(\nu-\nu_{0}\right)=\arg (\nu),
$$

the zero set of $L_{j}\left(\nu_{\circ}\right) f-\alpha$ is contained in

$$
S_{R}^{0}\left(\bigcup_{j=0}^{1} K(j n \nu), \pi / 2 n\right) \text {. }
$$

Proof. Let $\nu_{\circ}$ be a solution of $\arg \left(\boldsymbol{\nu}-\nu_{0}\right)=\arg (\boldsymbol{\nu})$ and $g(z)=$ $f(z)-\alpha$. By a theorem of Takagi [5, p. 83], the zero set of $g-\nu_{\circ} g^{\prime}$ is contained in $K_{1}=\mathscr{H}\left[\bigcup_{j=0}^{1} K\left(j n \nu_{\circ}\right)\right]$. Let us consider

$$
L_{1}\left(\nu_{\circ}\right) g=\left(g-\nu_{\circ} g^{\prime}\right)+\nu_{\circ}\left(g-\nu_{\circ} g^{\prime}\right)^{\prime} \text {. }
$$

As $L_{1}\left(\nu_{0}\right) g$ is a linear combination of a polynomial of degree $n$ and its first derivative, Takagi's theorem implies that its zero set lies in $K_{2}=\mathscr{H}\left[\bigcup_{j=0}^{1} K_{1}\left(-j n \nu_{\circ}\right)\right]$. But $K_{2} \subset S_{R}^{0}\left(K_{1}, \pi / 2 n\right)$. Let us assume that the zero set of $L_{j}\left(\nu_{\circ}\right) g$ lies in $K_{j+1}=\mathscr{H}\left[\bigcup_{m=0}^{1} K_{j}\left(-m n \nu_{\circ}\right)\right]$ and that $K_{j+1} \subset S_{R}^{0}\left(K_{1}, \pi / 2 n\right)$. Consider

$$
L_{j+1}\left(\nu_{\circ}\right) g=\left(g-\nu_{\circ}^{2 j} g^{\left(2^{j}\right)}\right)+\nu_{\circ}^{2 j}\left(g-\nu_{\circ}^{2^{j}} g^{\left(2^{j}\right)}\right)^{\left(2^{j}\right)} .
$$

The operator $L_{j+1}\left(\nu_{\circ}\right) g$ is a linear combination of an $n$th degree polynomial and its $2^{j}$ th derivative. By assumption the zero set of $g-$ $\nu_{\circ}^{2^{j}} g^{\left(2^{j}\right)}$ is in $S_{R}^{0}\left(K_{1}, \pi / 2 n\right)$. Applying this assumption to $L_{j+1}\left(\nu_{\circ}\right) h$ with $h=g-\nu_{\circ}^{2^{j}} g^{\left(2^{j}\right)}$, we find that the zero set of $L_{j+1}\left(\nu_{\circ}\right) g$ is in $S_{R}^{0}\left(K_{1}, \pi / 2 n\right)$ as was to be proved. The following observation completes the proof

$$
S_{R}^{0}\left(K_{1}, \pi / 2 n\right) \subset S_{R}^{0}\left(\bigcup_{j=0}^{1} K(j n \nu), \pi / 2 n\right) .
$$

We shall now consider the following application of this lemma.

THEOREM 2. Let $\alpha \in C, H$ be an AHP of degree $n$ and $K$ the convex hull of the $\alpha$ points of its associate. Let

$$
\mathscr{L}_{j}(\nu) H=H-\nu^{\nu^{j}}\left(\frac{\partial}{\partial x}\right)^{2^{j}} H, 0 \leqq j \leqq k
$$

where $k$ is the largest integer such that $2^{k} \leqq n$ and

$$
\nu \in S_{R}(K, \pi / 2 n) \cap S_{0}\left(\pi /(2 n)^{2}\right) .
$$

Then for any solution $\nu_{\circ}$ to the equation $\arg \left(\nu-\nu_{0}\right)=\arg (\nu)$,

$$
\mathscr{L}_{s}\left(\nu_{\circ}\right) H \neq \alpha
$$

in $C_{R}\left(\bigcup_{j=0}^{1} K(j n \nu), \pi / 2 n\right)$ for $0 \leqq s \leqq k$.

Proof. Let $h$ be the associate of $H$ and $g=h-\alpha$. Then 


$$
\mathscr{L}_{s}\left(\nu_{\circ}\right) H-\alpha=\frac{1}{2 \pi} \int_{0}^{2 \pi}\left\{g(u)-\nu_{\circ}^{2^{s}} g^{\left(2^{8}\right)}(u)\right\} d t
$$

with $u=x+i \rho$ cost. Let $l(z)=g(z)-\nu_{\circ}^{2^{s}} g^{\left(2^{s}\right)}(z)$ and factor $l(z)$,

$$
l(z)=A_{n}\left(\nu_{\circ}^{2^{s}}\right) \prod_{m=1}^{n}\left(z-\zeta_{m}\left(\nu_{\circ}^{2^{s}}\right)\right)
$$

By Lemma 1,

$$
\left\{\zeta_{m}\left(\nu_{\circ}^{28}\right)\right\} \bigcup_{m=1}^{n} \subset S_{R}^{0}\left(\bigcup_{j=0}^{1} K(j n \nu), \pi / 2 n\right) .
$$

If $x=x_{1}$ and $\rho=\rho_{1}$ are the equations of a circle in $C_{R}\left(\bigcup_{m=0}^{1} K(m n \nu)\right.$, $\pi / 2 n)$, then for $0 \leqq t \leqq 2 \pi$ the vector

$$
u_{1}(t)=x_{1}+i \rho_{1} \operatorname{cost} \in S_{R}\left(\bigcup_{j=0}^{1} K(j n \nu), \pi / 2 n\right) .
$$

By considering

$$
\frac{1}{2 \pi} \int_{0}^{2 \pi} l\left(u_{1}(t)\right) d t
$$

as the limit of a sum of vectors terminating in an open half plane with boundary through the origin, as in Theorem 1, we find that the integral cannot vanish, and thus

$$
\mathscr{L}_{s}\left(\nu_{\circ}\right) H \neq \alpha, 0 \leqq s \leqq k,
$$

in $C_{R}\left(\bigcup_{m=0}^{1} K(m n \nu), \pi / 2 n\right)$ as was to be proved.

We remark that a similar result holds for left zero free cones.

COROLLARY 2.1. Under the hypothesis of Theorem 2, for each solution $\nu_{\circ}$ of $\arg \left(\nu-\nu_{0}\right)=\arg (\nu)$,

$$
\mathscr{L}_{s}\left(\nu_{\circ}\right) \frac{\partial^{k}(H-\alpha)}{\partial x^{k}} \neq 0, \quad 1 \leqq s \leqq w
$$

in $C_{R}\left(\bigcup_{m=0}^{1} K(m n \nu), \pi / 2 n\right)$ where $k \leqq n-1$ and $w$ is the largest integer such that $2^{w} \leqq n-k$.

Proof. We need only observe that the zeros of the associate of $\partial^{k}(H-\alpha) / \partial x^{k}$ are in the convex hull of the zeros of the associate of $H-\alpha$ and apply Theorem 2 . We shall consider the following application of Lemma 1 .

Lemma 2. Let $\alpha \in C, f$ be a polynomial of degree $n$ and $K$ the convex hull of the $\alpha$ points of $f$. Let 


$$
G_{j}(\nu) f=f-\nu f^{(1)}+\nu^{2} f^{(2)}-\cdots+(-1)^{2 j+1} \nu^{2^{j}-1} f^{\left(2^{j}-1\right)}
$$

for $1 \leqq j \leqq k$ where $k$ is the largest integer such that $2^{k} \leqq n$ and $\nu \in$ $S_{R}(K, \pi / 2 n) \cap S_{0}\left(\pi /(2 n)^{2}\right)$. Then for every solution $\nu_{0}$ of the equation $\arg \left(\nu-\nu_{0}\right)=\arg (\nu)$, the zero set of $G_{s}\left(\nu_{0}\right) f-\alpha$ lies in $S_{R}^{0}\left(\bigcup_{m=0}^{1} K(m n \nu)\right.$, $\pi / 2 n)$ for $1 \leqq s \leqq k$.

Proof. If $s=1$, the result holds by Takagi's theorem cited in Lemma 1. For $s=2$ with $h=f-\alpha$

$$
\begin{gathered}
G_{2}\left(\nu_{\circ}\right) f-\alpha=h-\nu_{\circ} h^{(1)}+\nu_{\circ}^{2(2)}-\nu_{\circ}^{3} h^{(3)} \\
=\left(h-\nu_{\circ} h^{(1)}\right)+\nu_{\circ}^{2}\left(h-\nu_{\circ} h^{(1)}\right)^{(2)} .
\end{gathered}
$$

We set $g=h-\nu_{0} h^{(1)}$ and apply Lemma 1 to find that the zero set of $G_{2}\left(\nu_{\circ}\right) f-\alpha$ is in $S_{R}^{0}\left(\bigcup_{j=0}^{1} K(j n \nu), \pi / 2 n\right)$. Assume that the zero set of $g_{l}=G_{l-1}\left(\nu_{\circ}\right) f-\alpha$ is contained in $S_{R}^{0}\left(\bigcup_{j=0}^{1} K(j n \nu), \pi / 2 n\right)$. The proof is completed by noting that by assumption the zero set of

$$
g_{l-1}(z)=h(z)-\nu_{\circ} h^{(1)}(z)+\cdots+(-1)^{2 l-1}\left(\nu_{\circ}\right)^{2^{l-1}-1} h^{\left(2^{l-1}-1\right)}(z)
$$

is in $S_{R}^{\circ}\left(\bigcup_{j=0}^{1} K(j n \nu), \pi / 2 n\right)$ and applying Lemma 1 with

$$
\left.G_{l}\left(\nu_{\circ}\right) f-\alpha=g_{\ell-1}+\nu_{\circ}^{2^{l-1}}\left(g_{\ell-1}\right)^{\left(2^{l-1}\right)}\right)
$$

to find that the zero set of $G_{l}(\nu) f-\alpha$ is in

$$
S_{R}^{0}\left(\bigcup_{j=0}^{1} K(j n \nu), \pi / 2 n\right)
$$

The following theorem can be formulated in terms of left zero free cones. This formulation is left to the reader.

THEOREM 3. Let $\alpha \in C, H$ be an AHP of degree $n$ and $K$ denote the convex hull of the set of points for which its associate assumes the value $a$. Let

$$
\mathscr{Y}_{j}(\nu) H=H-\nu \frac{\partial H}{\partial x}+\nu^{2} \frac{\partial^{2} H}{\partial x^{2}}-\cdots+(-1)^{2 j+1} \nu^{2^{j}-1} \frac{\partial^{2 j-1} H}{\partial x^{2 j-1}},
$$

for $1 \leqq j \leqq k$ where $k$ is the largest integer such that $2^{k} \leqq n$ and $\nu \in S_{R}(K, \pi / 2 n) \cap S_{0}\left(\pi /(2 n)^{2}\right)$. Then for each solution $\nu_{0}$ if the equation $\arg \left(\nu-\nu_{0}\right)=\arg (\nu)$ and for $1 \leqq j \leqq k$,

$$
\mathscr{C}_{j}\left(\nu_{\circ}\right) H \neq \alpha,
$$

in $C_{R}\left(\bigcup_{m=n}^{1} K(m n \nu), \pi / 2 n\right)$.

Proof. The proof of this theorem uses Lemma 2 in a manner analogous to that of the use of Lemma 1 in Theorem 2. 
COROLLARY 3.1. Under the hypothesis of Theorem 3, for each solution $\nu_{0}$ of $\arg \left(\nu-\nu_{0}\right)=\arg (\nu)$,

$$
\mathscr{G}_{s}\left(\nu_{\circ}\right) \frac{\partial^{k}}{\partial x^{k}}(H-\alpha) \neq 0 \quad \text { with } \quad 1 \leqq s+k \leqq n
$$

in $C_{R}\left(\bigcup_{j=0}^{1} K(n j \nu), \pi / 2 n\right)$.

Proof. This result is proved in the same way as Corollary 2.1.

We shall now consider an AHP analog to the theorem of Lucas [4, p. 22]. One can easily show that if $H$ is an AHP and $K$ is the convex hull of the zero set of its associate, then the cones $C_{R}(K, \pi / 2 n)$ and $C_{L}(K, \pi / 2 n)$ are zero free cones for $\partial^{j} H / \partial x^{j}$ with $1 \leqq j \leqq n$. This provides an analog of the classical Lucas theorem which states that the convex hull of the zero set of a polynomial contains the zeros of its derivatives.

A sharper analog to the Lucas theorem will be drawn through the following observation. If $\Gamma_{0}$ denotes the convex hull of the zero set of an $n$th degree polynomial of one complex variable and $\Gamma_{k}$ denotes the convex hull of the zero set of its $k$ th derivative, Lucas theorem implies that $\Gamma_{0} \supseteqq \Gamma_{1} \supseteqq \cdots \supseteqq \Gamma_{n}$. Let $\widetilde{\Gamma}_{k}$ denote the complement of $\Gamma_{k}$, then $\widetilde{\Gamma}_{0} \subseteq \widetilde{\Gamma}_{1} \ldots \subseteq \widetilde{\Gamma}_{n}$ and the $k$ th derivative of the polynomial omits zero in $\widetilde{\Gamma}_{k}$.

Given a AHP of degree $n$, we shall construct a sequence of cones $\left\{C_{k}\right\}_{k=0}^{n}$ such that $C_{n} \supseteqq C_{n-1} \supseteqq \cdots \supseteqq C_{0}$ and such that the $j$ th derivative of the AHP with respect to $x$ has no zeros in $C_{j}$.

Theorem 4. Let

$$
H(x, \rho)=\sum_{k=j}^{n} a_{k} r^{k} P_{k}(\cos \theta)
$$

and

$$
\begin{aligned}
& \frac{\partial^{j} H}{\partial x^{j}}=\sum_{k=j}^{n} \frac{k !}{(k-j) !} a_{k} r^{k-j} P_{k-j}(\cos \theta) . \\
& 0 \leqq j \leqq n-1 .
\end{aligned}
$$

Let

$$
K_{j}=\left\{z \in C|| z\left|<1+\max _{j \leqq k \leqq n-1}\right| \frac{a_{k}}{a_{n}} \frac{k !(n-j) !}{n !(k-j) !} \mid\right\}, 0 \leqq j \leqq n-1
$$

Then if $x=x_{0}$ and $\rho=\rho_{0}$ are the equations of a circle in $C\left(K_{j}, n\right)=$ $C_{L}\left(K_{j}, \pi / 2 n\right) \cap C_{R}\left(K_{j}, \pi / 2 n\right)$, 
AXISYMMETRIC HARMONIC POLYNOMIALS AND THEIR DERIVATIVES 449

$$
\frac{\partial^{j} H}{\partial x^{j}}\left(x_{0}, \rho_{0}\right) \neq 0, \quad 0 \leqq j \leqq n-1
$$

and

$$
C\left(K_{j}, n\right) \leqq C\left(K_{j+1}, n\right), \quad 0 \leqq j \leqq n-1 .
$$

Proof. A theorem due to Marden [4, p. 140], implies that $H(x$, $\rho) \neq 0$ in $C\left(K_{0}, n\right)$. Applying this result to $\partial^{j} H / \partial x^{j}$, we find that $\partial^{j} H / \partial x^{j} \neq 0$ in $C\left(K_{j}, n\right)$ as was to be proved. Note that

$$
\max _{j \leqq k \leqq n-1}\left|\frac{a_{k}}{a_{n}} \frac{k !}{n !} \frac{(n-j) !}{(k-j) !}\right| \geqq \max _{j+1 \leqq k \leqq n-1}\left|\frac{a_{k}}{a_{n}} \frac{k !}{n !} \frac{(n-j-1) !}{(k-j-1) !}\right|
$$

with strict inequality holding if $a_{k} \neq 0$ for some $k \geqq j$.

This implies that $K_{j} \supseteqq K_{j+1}$ and thus $C\left(K_{j}, n\right) \subseteq C\left(K_{j+1}, n\right)$ as was to be proved.

We shall now consider extensions to generalized axisymmetric harmonic polynomials (GAHP). Let $\left(x_{1}, \cdots, x_{n}\right)$ be a point in $E^{n}$ and $(x, \rho)$ be generalized cylindrical coordinates with $\rho^{2}=x_{2}^{2}+\cdots+x_{n}^{2}$. R. P. Gilbert shows [2, p. 168] that GAHP $U(x, \rho)$ may be generated by the operator

$$
U(x, \rho)=A_{\mu} \int_{0}^{\pi} u(x+i \rho \cos t)(\sin t)^{2 \mu-1} d t
$$

with, $2 \mu=n-2, A_{\mu}=\Gamma(\mu-1 / 2) /\left(\pi^{1 / 2} \Gamma(\mu)\right)$ and $u(\zeta)$ a polynomial of degree $n, \zeta \in C$. Let the cones described by equations (1) and (2) be interpreted with $\rho^{2}=x_{2}^{2}+\cdots+x_{n}^{2}$. As $(\sin t)^{2 \mu-1}>0$ for $0<t<\pi$, the proofs of Theorems 1 through 3 are basically unchanged. We cite the following result as a typical generalization.

THEOREM 5. Let $\alpha \in C, G$ be a GAHP having a polynomial of degree $n$ as its associate. Let $K$ denote the convex hull of the $\alpha$ points of the associate. Then for each

$$
\left\{\lambda_{1}, \cdots, \lambda_{p}\right\} \subset S_{L}(K, \pi / 2 n) \cap S_{\pi}(\pi / 2 n) \text { with } 1 \leqq p \leqq n,
$$

the GAHP

$$
\left(1-\lambda_{1} \frac{\partial}{\partial x}\right) \cdots\left(1-\lambda_{p} \frac{\partial}{\partial x}\right) G
$$

omits $\alpha A_{\mu} \beta_{\mu}$ in $C_{R}(K, \pi / 2 n)$. For each $\left\{\mu_{1}, \cdots, \mu_{p}\right\} \subset S_{R}(K, \pi / 2 n) \cap$ $S_{0}(\pi / 2 n)$, the $G A H P$

$$
\left(1-\mu_{1} \frac{\partial}{\partial x}\right) \cdots\left(1-\mu_{p} \frac{\partial}{\partial x}\right) G
$$


omits $\alpha A_{\mu} \beta_{\mu}$ in $C_{L}(K, \pi / 2 n)$ with $\beta_{\mu}=\int_{0}^{\pi}(\sin t)^{2 \mu-1} d t$.

It is clear that Theorem 4 has its corresponding extension to GAHP with Gilbert's operator. We note that the linear combinations of AHP and GAHP considered in this paper are themselves harmonic. If corresponding results are considered for the linear combinations of $\mathrm{AHP}$ and GAHP with respect to the radial derivatives, the resulting linear combinations are no longer harmonic. Also, we point out that most of the preceding theorems will cover AHP of degree one by suitable modifications in the angles described.

\section{REFERENCES}

1. Stefan Bergman, Integral Operators in the Theory of Linear Partial Differential Equation, Ergebnisse der Mathematik und ihrer Grenzgebiete, N. F., Heft 23, SpringerVerlag, Berlin, 1961.

2. R. P. Gilbert, Function Theoretic Methods in Partial Differential Equations, Math. in Science and Engineering, vol. 54, Academic Press, New York, 1969.

3. O. D. Kellog, Foundations of Potential Theory, Springer-Verlag, Berlin, 1929.

4. Morris Marden, Value distribution of harmonic polynomials in several real variables, Trans. Amer. Math. Soc., 159 (1971).

5. — Geometry of Polynomials, Math. Surveys, no. 3, Providence, R. I., 1966.

Received July 11, 1972.

Mathematics Department

U. S. Naval ACademy, ANnapolis, Maryland, 21402 